\title{
Bad to the bone
}

Evidence that suppressing the hedgehog $(\mathrm{Hh})$ signalling pathway in cancers has potential therapeutic value has led to the development of drugs that target this pathway. However, results from Tom Curran and colleagues indicate that suppressing Hh signalling in young mice results in permanent and detrimental skeletal changes.

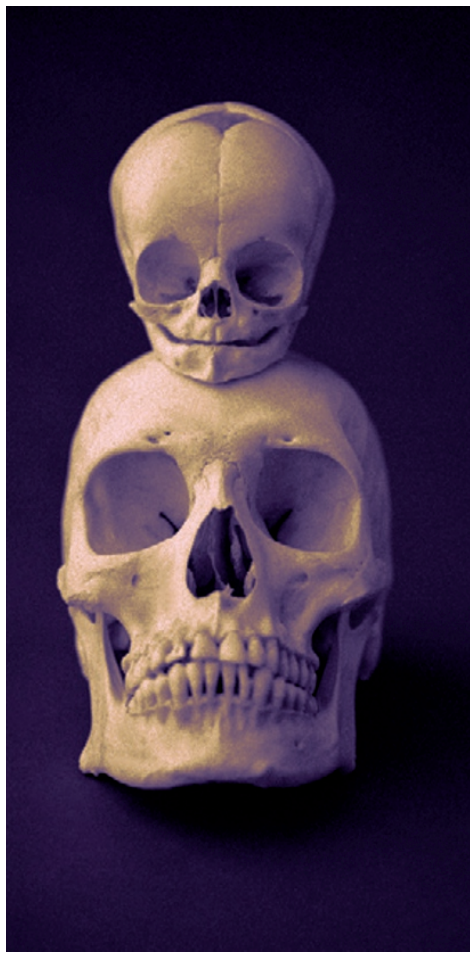

Mutation of Hh pathway genes, such as $\underline{P T C H 1}$ (patched 1) and $\underline{S M O}$ (smoothened), are found in basal cell carcinoma and the predominantly paediatric tumour medulloblastoma. To enable functional imaging of the Hh pathway in mice, Curran and colleagues generated reporter GliLuc mice. Gli is a downstream target of SMO and is known to be an effective reporter of Hh activity when fused to the firefly luciferase gene. When treated with HhAntag, an inhibitor of SMO, at doses ranging between $10-100 \mathrm{mg} / \mathrm{kg}$, postnatal day 10 GliLuc transgenic mice showed reduced GLI activity in the skin and brain, and this was maintained for a total of eight doses over 4 days. The pathway was active again 2 days after the drug was stopped.

Having previously shown that in adult mouse models of medulloblastoma 2 weeks of treatment with twice daily $100 \mathrm{mg} / \mathrm{kg}$ HhAntag had therapeutic efficacy and no obvious toxicity, the authors were surprised to find that the young mice treated with the drug failed to thrive and remained smaller than control mice. $\mathrm{X}$-ray analyses showed that these treated mice had widespread skeletal changes, including gross abnormalities of the tibia and femur. Further analyses showed that HhAntag blocks proliferation of chondrocytes (which produce cartilage) and promotes their differentiation. After HhAntag withdrawal, osteogenesis and bone remodelling were evident, as was increased deposition of bone minerals within the growth plate of the long bones. All these findings suggest that HhAntag treatment leads to premature fusion of the growth plate and permanent bone defects

The authors were able to show that no abnormalities arose in mice treated with $10 \mathrm{mg} / \mathrm{kg}$ HhAntag, but once the dose was increased to $25 \mathrm{mg} / \mathrm{kg}$ the skeletal defects were apparent. Unfortunately, the GliLuc data indicate that a dose of $10 \mathrm{mg} / \mathrm{kg}$ is not sufficient to completely block Hh activity, and this dose is not effective for tumour eradication in adult mice. Therefore, although $\mathrm{Hh}$ antagonists might be efficacious for medulloblastoma treatment, their systemic use in young children will need to be carefully monitored. Moreover, these findings argue that new molecular targeted therapies with potential use in children should be investigated for developmental effects in young mice.

Nicola McCarthy

ORIGINAL RESEARCH PAPER Kimura, H., Ng J. M. Y. \& Curran, T. Transient inhibition of the hedgehog pathway in young mice causes permanent defects in bone structure. Cancer $\mathrm{Cell}$ 13, 249-260 (2008) 